\title{
Clinical and genetic analysis of the first known Asian family with myotonic dystrophy type 2
}

\author{
Takahiro Nakayama ${ }^{1}$, Harumasa Nakamura ${ }^{2}$, Yasushi Oya ${ }^{2}$, Takashi Kimura ${ }^{3}$, Ichiro Imahuku ${ }^{1}$, Kinji Ohno ${ }^{4}$, \\ Ichizo Nishino ${ }^{5}$, Koji Abe $^{6}$ and Tohru Matsuura ${ }^{6,7}$
}

Myotonic dystrophy type 2 (DM2) is more common than DM1 in Europe and is considered a rare cause of myotonic dystrophies in Asia. Its clinical course is also milder with more phenotypic variability than DM1. We herein describe the first known Asian family (three affected siblings) with DM2 based on clinical and genetic analyses. Notably, two of the affected siblings were previously diagnosed with limb-girdle muscular dystrophy. Myotonia (the inability of the muscle to relax) was absent or only faintly present in these individuals. The third sibling had grip myotonia and is the first known Asian DM2 patient. The three DM2 siblings share several systemic characteristics, including late-onset, proximal-dominant muscle weakness, diabetes, cataracts and asthma. Repeat-primed PCR across the DM2 repeat revealed a characteristic ladder pattern of a CCTG expansion in all siblings. Southern blotting analysis identified the presence of 3400 repeats. Further DM2 studies in Asian populations are needed to define the clinical presentation of Asian DM2 and as yet unidentified phenotypic differences from Caucasian patients.

Journal of Human Genetics (2014) 59, 129-133; doi:10.1038/jhg.2013.133; published online 16 January 2014

Keywords: Asian; CCTG repeat; clinical spectrum; haplotype; limb-girdle muscular dystrophy; myotonia; myotonic dystrophy type 2 (DM2)

\section{INTRODUCTION}

Myotonic dystrophy (DM) is the most common adult-onset muscular dystrophy and is characterized by autosomal dominant progressive myopathy (muscle weakness), myotonia (the inability of the muscle to relax) and multiorgan involvement. Two genetically distinct forms of the disease with clinical similarities but distinct differences are known: DM type 1 (DM1) and type 2 (DM2). DM1 is caused by the expansion of a CTG repeat in the $3^{\prime}$-untranslated region of the dystrophia myotonica-protein kinase gene on chromosome $19 \mathrm{p} 13.3,{ }^{1}$ while DM2 is caused by an expansion of a tetranucleotide CCTG repeat in the first intron of the cellular nucleic acid-binding protein $(C N B P$, formerly ZNF9) gene on chromosome $3 \mathrm{q} 21 .^{2}$ The unprecedented number of expanded DM2 CCTG repeats (ranging from 75 to 11000 with a mean of 5000) is highly unstable in intergenerational transmissions and varies in a tissue-specific, time-dependent manner. ${ }^{2}$

The clinical severity and spectrum of DM2 is highly variable, and this is not thought to be associated with the length of repeat expansions. ${ }^{2,3}$ Most DM2 mutations have been identified in European Caucasians that originate from a single common founder and share an identical haplotype ${ }^{4-6}$ However, we previously identified the first Japanese DM2 patient carrying a haplotype distinct from that shared among Caucasians, indicating that DM2 exists in nonCaucasian populations and has separate founders. ${ }^{7}$ Thus, it would be beneficial to investigate whether DM2 patients of different ethnicities and haplotypes have a comparable phenotype to the predominantly European patients with a common haplotype. To further characterize the variable clinical phenotype of DM2, we describe the clinical and molecular findings of the first known Asian family with DM2, including the previously reported Japanese female patient ${ }^{7}$ and her two affected siblings.

\section{SUBJECTS AND METHODS}

The pedigree studied is shown in Figure 1. DNA was extracted from peripheral blood samples obtained from the three affected family members with their informed consent. Approval for the study was obtained from the ethics committees of Okayama University, Nagoya University Graduate School of Medicine and the National Center of Neurology and Psychiatry.

PCR products across the DM2 repeat (marker CL3N58) ${ }^{2}$ in the first intron of CNBP were analyzed by capillary electrophoresis using an automated DNA sequencer (ABI 310A Genetic Analyzer, Applied Biosystems, Foster City, CA, USA). The PCR reaction was performed in a $20 \mu \mathrm{l}$ volume containing $100 \mathrm{ng}$ genomic DNA as template, $1 \times$ HotStarTaq Plus Master Mix (Qiagen, Valencia, CA, USA) and $0.2 \mu \mathrm{M}$ each of the primers: 6FAM-fluorescent-labeled

\footnotetext{
${ }^{1}$ Department of Neurology, Yokohama Rosai Hospital, Yokohama, Japan; ${ }^{2}$ Department of Neurology, National Center Hospital, National Center of Neurology and Psychiatry, Tokyo, Japan; ${ }^{3}$ Department of Neurology, Asahikawa Medical Center, National Hospital Organization, Asahikawa, Japan; ${ }^{4}$ Division of Neurogenetics, Center for Neurological Diseases and Cancer, Nagoya University Graduate School of Medicine, Nagoya, Japan; ${ }^{5}$ Department of Neuromuscular Research, National Institute of Neuroscience, National Center of Neurology and Psychiatry, Tokyo, Japan; ${ }^{6}$ Department of Neurology, Okayama University Graduate School of Medicine, Dentistry and Pharmaceutical Sciences, Okayama, Japan and 7 Division of Neurology, Department of Medicine, Jichi Medical University, Shimotsuke, Japan

Correspondence: Dr T Matsuura, Division of Neurology, Department of Medicine, Jichi Medical University, 3311-1 Yakushiji, Shimotsuke, Tochigi 329-0498, Japan. E-mail:tohrum@jichi.ac.jp
}

Received 20 March 2013; revised 16 November 2013; accepted 4 December 2013; published online 16 January 2014 
CL3N58-D F (5'-GCCTAGGGGACAAAGTGAGA-3') and CL3N58-D R (5'-GGCCTTATAACCATGCAAATG- $3^{\prime}$ ). The PCR conditions consisted of an initial denaturation at $95^{\circ} \mathrm{C}$ for $5 \mathrm{~min}$, then 30 cycles of $94^{\circ} \mathrm{C}$ for $30 \mathrm{~s}, 57^{\circ} \mathrm{C}$ for $30 \mathrm{~s}$ and $72{ }^{\circ} \mathrm{C}$ for $30 \mathrm{~s}$, with an additional extension at $72{ }^{\circ} \mathrm{C}$ for $10 \mathrm{~min}$.

To detect the DM2 CCTG expansion, the repeat-primed PCR assay using an oligonucleotide primed within the DM2 CCTG repeat and Southern blotting analysis were performed as described elsewhere. ${ }^{2,3,7,8}$ Briefly, repeat-primed $\mathrm{PCR}^{8}$ was performed in a $20 \mu \mathrm{l}$ volume containing $100 \mathrm{ng}$ genomic DNA as template, $1 \times$ HotStarTaq Plus Master Mix (Qiagen), $1 \mathrm{~m}$ betaine, $0.2 \mu \mathrm{m}$ 6FAM-fluorescent-labeled CL3N58-D $\quad$ F $\quad$ (5'-GCCTAGGGGACAAAGTG AGA-3 $\left.3^{\prime}\right), 0.05 \mu \mathrm{M}$ reverse primer consisting of five CCTG repeats with an anchor tail: DM2-CCTG-for (5'-AGCGGATAACAATTTCACACAGGACCTGC CTGCCTGCCTGCCTG- $3^{\prime}$ ) and $0.3 \mu \mathrm{m}$ anchor primer corresponding to the anchor tail of the reverse primer: P3 (5'-AGCGGATAACAATTTCACACA GGA-3 $3^{\prime}$ ). The PCR conditions were as follows: initial denaturation at $95^{\circ} \mathrm{C}$ for 5 min, then 35 cycles of $94^{\circ} \mathrm{C}$ for $1 \mathrm{~min}, 61^{\circ} \mathrm{C}$ for $1 \mathrm{~min}$ and $72^{\circ} \mathrm{C}$ for $1 \mathrm{~min}$, with an additional extension at $72{ }^{\circ} \mathrm{C}$ for $10 \mathrm{~min}$. Fragment length analysis was performed on an ABI 310A Genetic Analyzer. Southern blotting was carried out with EcoRI-digested DNA $(10 \mu \mathrm{g})$ separated on an $0.8 \%$ agarose gel, which was transferred to a positively charged nylon membranes (Roche, Indianapolis, IN, USA) and hybridized with a 474-bp CNBP probe. $^{2}$

\section{RESULTS}

\section{Genetic studies}

Three patients with DM2 CCTG expansion were identified in this pedigree. Case 3 has been reported previously. ${ }^{7}$ All cases showed one peak following PCR of the DM2 repeat and a characteristic ladder pattern by repeat-primed PCR, ${ }^{3,7}$ confirming the presence of the CCTG expansion (Figure 2). DNA extracted from the Case 2 individual was degraded, so Southern blotting hybridization analysis was performed on the remaining two patients (Cases 1 and 3). This

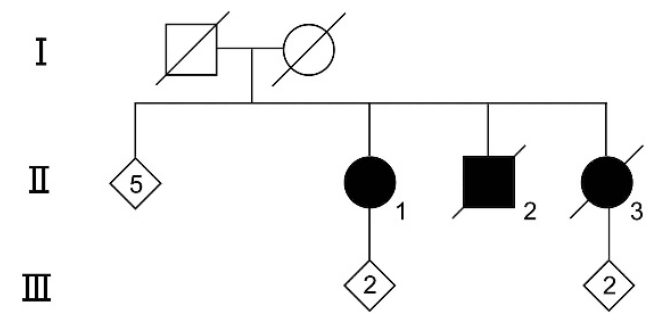

Figure 1 Pedigree of the sibling cases carrying the DM2 expansion. The parents did not suffer from muscle weakness and died at the ages of 67 and 72 years. The gender of the unaffected siblings and children of the affected cases is obscured to protect privacy. showed the presence of an $18.1 \mathrm{~kb}$ expanded DM2 allele, corresponding to 3400 CCTG repeats (Figure 3).

\section{Case reports}

Table 1 summarizes the clinical manifestations of three symptomatic siblings carrying the DM2 mutation. The siblings share common clinical features, including adult-onset proximal dominant progressive weakness, cataracts, diabetes, cardiac arrhythmias and hypercholesteremia, as described in the previous literature on DM2. ${ }^{7}$ The degree of myotonia (the cardinal feature of DM) is variable, with two siblings having little or no myotonia. Interestingly, all three were asthmatic. The data of allergic and autoimmune tests are shown in Table 2. The other asymptomatic members of the pedigree were not assessed.

\section{Case 1}

A 69-year-old Japanese woman first presented at the age of 64 years with a slowly progressive difficulty in walking. Her calves had become

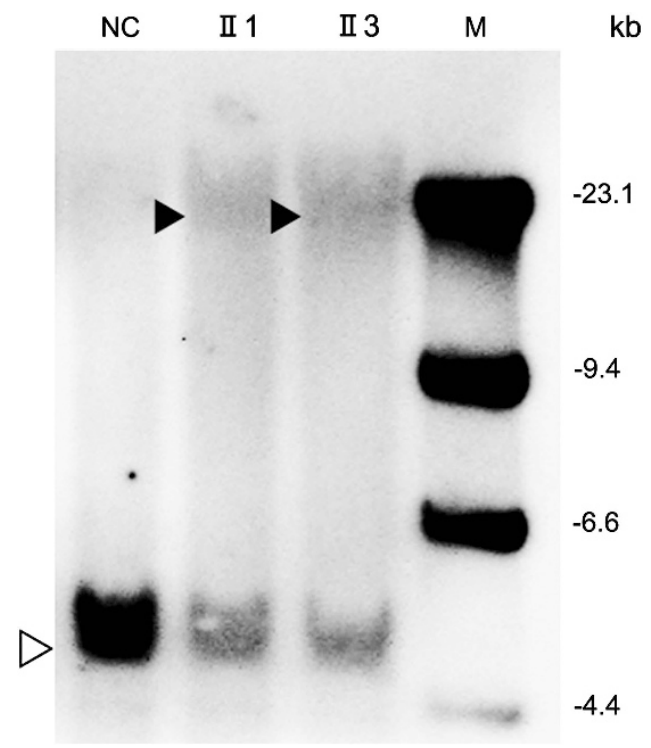

Figure 3 Southern blotting analysis of DM2. Closed arrowhead shows the expanded alleles in DM2. M, גDNA/Hind III marker; NC, normal control; III and $I I 3$, Cases 1 and 3 showing an 18.1-kb expanded allele as well as a normal allele (open arrowhead).

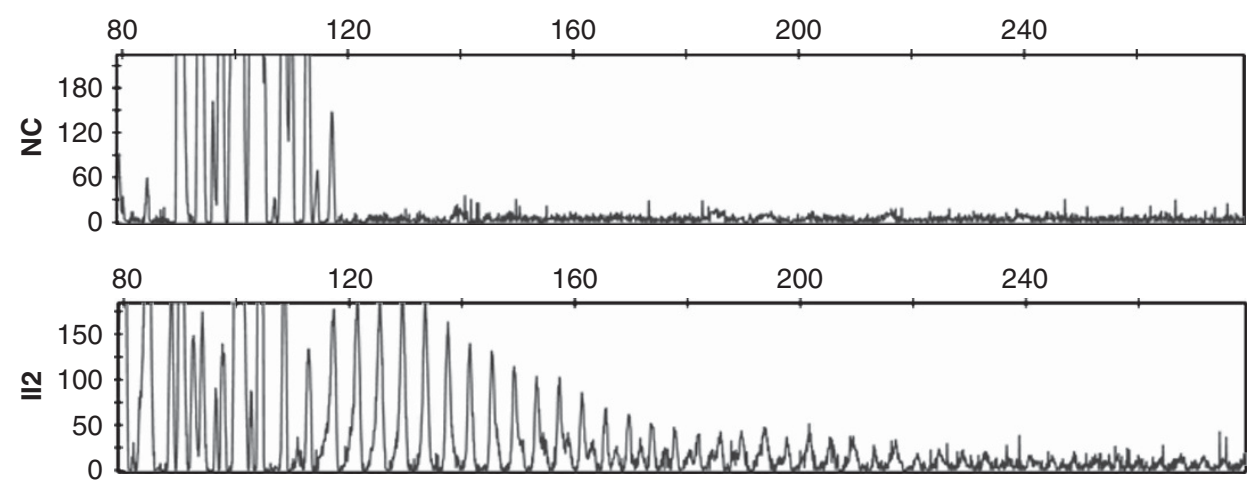

Figure 2 Repeat-primed PCR analysis specific for the DM2 expansion. Negative results from normal control (NC) are shown in the upper panel, whereas a characteristic continuous ladder from Case 2 (II2 in Figure 1), indicating the CCTG expansion, is detected in the lower panel. A full color version of this figure is available at the Journal of Human Genetics journal online. 
Table 1 Clinical features of DM2 patients in the Japanese pedigree

\begin{tabular}{|c|c|c|c|}
\hline \multirow[b]{2}{*}{ Characteristic } & \multicolumn{3}{|c|}{ DM2 individuals } \\
\hline & Case 1 & Case 2 & Case $3^{7}$ \\
\hline Age at DM2 onset (years) & 64 & 50 & 47 \\
\hline \multicolumn{4}{|l|}{ Skeletal muscle features } \\
\hline EMG diagnosis & $-1+$ & - & + \\
\hline Myalgia/stiffness & + & $-1-$ & $-1-$ \\
\hline \multicolumn{4}{|l|}{ Muscle weakness/atrophy } \\
\hline Facial muscles & + & - & + \\
\hline Sternocleidomastoids & + & + & + \\
\hline Limbs & Proximal dominant & Proximal dominant & Proximal dominant \\
\hline \multicolumn{4}{|l|}{ Systemic features } \\
\hline ECG & Normal & Normal & cRBBB \\
\hline Holter monitoring & PVC & PVC & PVC \\
\hline \multirow[t]{3}{*}{ Other disorders } & Asthma, HT, low IgG & Asthma, HT & Asthma, low IgG \\
\hline & Hypercholesterolemia & Hypercholesterolemia & Hypercholesterolemia \\
\hline & Hyperthyroidism & & \\
\hline Initial clinical diagnosis & LGMD & LGMD & Myotonic dystrophy \\
\hline
\end{tabular}

Abbreviations: cRBBB, complete right bundle branch block; DM2, myotonic dystrophy type 2; EMG, electromyogram; ECG, electrocardiogram; F, female; HT, hypertension; IgG, immunoglobulin G; LGMD, limb-girdle muscular dystrophy; M, male; PVC, premature ventricular contraction.

Table 2 Profile of autoantibodies, IgE and eosinophils in this pedigree

\begin{tabular}{lccc}
\hline & \multicolumn{3}{c}{ DM2 individuals } \\
\cline { 2 - 4 } & Case 1 & Case 2 & Case 3 \\
\hline Antinuclear antiboby & Negative & Negative & Negative \\
Rheumatoid factor & Negative & Negative & Negative \\
Anti-thyroglobulin antibody & Positive & NE & Negative \\
TSH receptor antibody & Positive & NE & Negative \\
IgE (normal: $<250$ IU $\mathrm{ml}^{-1}$ ) & NE & 1700 & 52.8 \\
Eosinophils (normal: $0-5 \%$ of leukocytes) & $0-25$ & $10.4-13.9$ & 3
\end{tabular}

Abbreviations: DM2, myotonic dystrophy type 2; IgE, immunoglobulin E; NE, not examined; TSH, thyroid stimulating hormone.

stiff over the last 2 or 3 years. There was no history of developmental milestone delay. She had a past medical history of taking asthma and hyperthyroidism medication. At 33 years of age, she had developed type 2 diabetes mellitus and hypertension. At 59 years, she had had both posterior subcapsular cataracts extracted. There was no complaint of myalgia. There was no known consanguinity or genetic admixture with other ethnicities in her family, as described elsewhere. ${ }^{7}$ Neither of her parents, her three older brothers, two older sisters nor either of her two children had been referred with evident muscle symptoms. On examination, she was able to walk upstairs by holding onto a rail and walking on flat ground. She showed mild facial weakness as well as weakness and atrophy of sternocleidomastoid muscles. Motor examination showed slight symmetric muscle atrophy and predominant proximal weakness.
Percussion myotonia could be slightly induced in the tongue and thenar eminence; however, grip myotonia was not present. Tendon reflexes were within the normal range. There were no cerebellar, sensory or autonomic disorders. Serum creatine kinase elevation was evident at $413 \mathrm{IUl}^{-1}$ (normal range: $37-115 \mathrm{IUl}^{-1}$ ), and high hemoglobin A1c $(7.6 \%)$ and total cholesterol levels $\left(248 \mathrm{mg} \mathrm{dl}^{-1}\right)$ were seen. The serum immunoglobulin $\mathrm{G}$ level was slightly decreased to $808 \mathrm{mg} \mathrm{dl}^{-1}$ (normal range: $870-1700 \mathrm{mg} \mathrm{dl}^{-1}$ ). Holter monitoring detected premature ventricular contractions. Electromyography showed small motor unit potentials with occasional myotonic discharges in all the muscles examined. Nerve conduction studies were normal. Muscle computed tomography revealed diffuse muscle atrophy in the trunk and proximally in all limbs, whereas forearm and distal leg muscles were well preserved. Fluid-attenuated inversion recovery and T2-weighted brain magnetic resonance imaging showed non-specific periventricular white matter hyperintensities without significant cerebral atrophy.

\section{Case 2}

A 61-year-old man, the younger brother of case 1, began to notice difficulty lifting his knees when climbing mountains or stairs at the age of 50 years. He had had a normal birth and development. At the age of 55 years, he had started to hold onto something when rising from a supine or sitting position and to feel difficulty in lifting his right arm. At 40 years of age, he had developed type 2 diabetes mellitus and hypertension. At 62 years, he had a left posterior subcapsular cataract extracted. He had a past medical history of taking asthma medication. He was referred to our hospital by his primary physician because of an elevated creatine kinase level of 
$1094 \mathrm{IUl}^{-1}$ (normal range: $<195 \mathrm{IU}^{-1}$ ). There was no complaint of muscle pain or stiffness.

Neurologically, the patient had normal language, speech and cognition on routine clinical evaluation. There were no cranial nerve abnormalities except for mild atrophy and weakness of the bilateral sternocleidomastoid muscles. Motor examination revealed predominant proximal muscle weakness and atrophy in all the limbs. In particular, atrophy of the right deltoid muscle and infraspinatus muscle and weakness of the right shoulder abduction and extortion were remarkable. There was no grip or percussion myotonia. Tendon reflexes were present but were hypoactive in both upper limbs. There were no cerebellar, sensory or autonomic disorders. There was slight serum creatine kinase elevation at $321 \mathrm{IUl}^{-1}$ (normal range: 62-287 $\mathrm{IU}^{-1}$ ) and high hemoglobin Alc (7.2\%) and total cholesterol levels $\left(271 \mathrm{mg} \mathrm{dl}^{-1}\right)$. The serum immunoglobulin $\mathrm{G}$ level was $915 \mathrm{mg} \mathrm{dl}^{-1}$ (normal range: $870-1700 \mathrm{mg} \mathrm{dl}^{-1}$ ). The electrocardiogram was normal, but 24-h Holter monitoring detected occasional premature ventricular contractions. Electromyography showed small motor unit potentials with early recruitment and complex repetitive discharges in all the muscles examined. No myotonic discharges were observed. Nerve conduction studies were normal. Muscle computed tomography revealed diffuse muscle atrophy in the trunk and proximally in all the limbs, whereas forearm and distal leg muscles were well preserved. The patient died from gastric cancer at the age of 66 years. Brain magnetic resonance imaging at the age of 65 years was normal without significant cerebral atrophy.

Case 3

The clinical presentation of Case 3 was previously described by Saito et al. ${ }^{7}$ In addition, the patient had a past medical history of bronchial asthma, and hypercholesterolemia was detected $\left(282 \mathrm{mg} \mathrm{dl}^{-1}\right)$ at the age of 59 years. The patient died suddenly at the age of 66 years of unknown causes.

\section{DISCUSSION}

This is the first known report of an Asian family with DM2. As expected for a dominantly inherited disorder, approximately $50 \%$ of first-degree relatives are at risk for DM2. However, given that clinical information is only available for three affected individuals among 13 members of a family of three consecutive generations, incomplete penetrance with asymptomatic carriers is the more favorable explanation of the pedigree. It could also reflect insufficient information about mild symptoms or signs in the past.

Notably, two of the affected members (Cases 1 and 2) had previously been diagnosed with limb-girdle muscular dystrophy because of the absence or subtlety of myotonia until their younger sister (Case 3) was genetically confirmed as the first Asian DM2 patient. Based on a report by Day et al., ${ }^{3} 90 \%$ of affected DM2 individuals from the European and American families have electrical myotonia and $75 \%$ have clinical myotonia. There is a possibility that Japanese/Asian DM2 individuals are underdiagnosed or misdiagnosed as another muscle disease because of the lack of myotonia ${ }^{9}$ and the previous conception of the rarity of DM2 in Asian populations. ${ }^{10}$ As seen in other repeat expansion disorders such as spinocerebellar ataxia type 2 (SCA2), Machado-Joseph disease/SCA3 and SCA10, ${ }^{11-13}$ we also have to keep in mind the clinical differences between different ethnicities and geographical regions, which cannot be explained by the repeat size. A unique Japanese/Asian haplotype mutation ${ }^{7}$ may also be related to low disease penetrance or the clinical presentation of mild DM2.

Multi-systemic features such as cataracts, diabetes, obesity, hypogammaglobulinemia, cardiac conduction block and arrhythmia are often seen in both DM1 and DM2 patients. ${ }^{3,14}$ In addition, a strong association between Dutch DM2 patients and autoimmune diseases or autoantibody production was recently reported, in contrast with those with DM1 whose data were comparable with the general population. ${ }^{15}$ Interestingly, all three patients in the present study were asthmatic, yet this association has not been previously described in the literature. To examine whether it is a true association or a mere coincidence, more families with different genetic backgrounds should be investigated. It is conceivable that the DM2 expansion directly affects the immune system or that the co-existence of a genetic change in flanking regions in linkage disequilibrium with the DM2 expansion confers susceptibility to allergic or autoimmune diseases.

It is well known that non-coding repeat expansion disorders, especially DM2, are associated with intra/inter-familial clinical variations and complex genotype-phenotype correlations. ${ }^{2,3}$ Therefore, to achieve an improved diagnosis of DM2, clinicians should examine as many family members as possible in the same pedigree and be aware of its wide clinical spectrum, which is possibly influenced by racial and environmental factors. Although this study could not determine characteristic features in Asian DM2, further collection of additional Asian DM2 families is needed to investigate clinical and genetic differences between Caucasian and Asian patients with DM2. Comparison of the association between phenotype and ethnic/ haplotype differences will be made possible in the future by the clinical and genetic assessment of DM2 subjects with different founders.

\section{CONFLICT OF INTEREST}

The authors declare no conflicts of interest.

\section{ACKNOWLEDGEMENTS}

We are grateful to the patients and their family for participating in this study. The study was supported by JSPS KAKENHI Grant Numbers 24390083, 23659455 (to TM), 20390250 (to IN), 21390267 (to KA) and 24390221 (to KO), as well as Research Grants (to TM, IN, KA and KO) for Intractable Diseases from the Ministry of Health, Labour and Welfare of Japan.

1 Harper, P. S. in Myotonic Dystrophy (W.B. Saunders, London, UK, 2001).

2 Liquori, C. L., Ricker, K., Moseley, M. L., Jacobsen, J. F., Kress, W., Naylor, S. L. et al. Myotonic dystrophy type 2 caused by a CCTG expansion in intron 1 of ZNF9. Science 293, 864-867 (2001).

3 Day, J. W., Ricker, K., Jacobsen, J. F., Rasmussen, L. J., Dick, K. A., Kress, W. et al. Myotonic dystrophy type 2: molecular, diagnostic and clinical spectrum. Neurology 60 , 657-664 (2003)

4 Liquori, C. L., Ikeda, Y., Weatherspoon, M., Ricker, K., Schoser, B. G., Dalton, J. C. et al. Myotonic dystrophy type 2: human founder haplotype and evolutionary conservation of the repeat tract. Am. J. Hum. Genet. 73, 849-862 (2003).

5 Bachinski, L. L., Udd, B., Meola, G., Sansone, V., Bassez, G., Eymard, B. et al Confirmation of the type 2 myotonic dystrophy (CCTG) $)_{n}$ expansion mutation in patients with proximal myotonic myopathy/proximal myotonic dystrophy of different European origins: a single shared haplotype indicates an ancestral founder effect. Am. J. Hum. Genet 73, 835-848 (2003).

6 Coenen, M. J., Tieleman, A. A., Schijvenaars, M. M., Leferink, M., Ranum, L. P. Scheffer, $\mathrm{H}$. et al. Dutch myotonic dystrophy type 2 patients and a North-African DM2 family carry the common European founder haplotype. Eur. J. Hum. Genet. 19, 567-570 (2011).

7 Saito, T., Amakusa, Y., Kimura, T., Yahara, O., Aizawa, H., Ikeda, Y. et al. Myotonic dystrophy type 2 in Japan: ancestral origin distinct from Caucasian families. Neurogenetics 9, 61-63 (2008). 
8 Radvansky, J., Ficek, A. \& Kadasi, L. Upgrading molecular diagnostics of myotonic dystrophies: multiplexing for simultaneous characterization of the DMPK and ZNF9 repeat motifs. Mol. Cell Probes 25, 182-185 (2011).

9 Young, N. P., Daube, J. R., Sorenson, E. J. \& Milone, M. Absent, unrecognized, and minimal myotonic discharges in myotonic dystrophy type 2. Muscle Nerve 41, 758-762 (2010).

10 Matsuura, T., Minami, N., Arahata, H., Ohno, K., Abe, K., Hayashi, Y. K. et al. Myotonic dystrophy type 2 is rare in the Japanese population. J. Hum. Genet. 57, 219-220 (2012).

11 Gwinn-Hardy, K., Chen, J. Y., Liu, H. C., Liu, T. Y., Boss, M., Seltzer, W. et al. Spinocerebellar ataxia type 2 with parkinsonism in ethnic Chinese. Neurology 55, 800-805 (2000).

12 Subramony, S. H., Hernande,z, D., Adam, A., Smith-Jefferson, S., Hussey, J., Gwinn-Hardy, K. et al. Ethnic differences in the expression of neurodegenerative disease: Machado-Joseph disease in Africans and Caucasians. Mov. Disord. 17, 1068-1071 (2002).
13 Teive, H. A., Roa, B. B., Raskin, S., Fang, P., Arruda, W. O., Neto, Y. C. et al. Clinical phenotype of Brazilian families with spinocerebellar ataxia 10. Neurology 63, 1509-1512 (2004).

14 Meola, G. \& Moxley, R. T. III Myotonic dystrophy type 2 and related myotonic disorders. J. Neurol. 252, 1173-1182 (2004).

15 Tieleman, A. A., den Broeder, A. A., van de Logt, A. E. \& van Engelen, B. G. Strong association between myotonic dystrophy type 2 and autoimmune diseases. J. Neurol. Neurosurg. Psychiatry 80, 1293-1295 (2009).

(c) (i) $(\Theta)$ This work is licensed under a Creative Commons Attribution-NonCommercial-NoDerivs 3.0 Unported License. To view a copy of this license, visit http://creativecommons. org/licenses/by-nc-nd/3.0/ 